\title{
ESTUDO QUÍMICO E PERFIL CROMATOGRÁFICO DAS CASCAS DE Aspidosperma parvifolium A. DC. ("PAU- PEREIRA")
}

Rose Lisieux R. Paiva Jácome* e Alaíde Braga de Oliveira

Departamento de Produtos Farmacêuticos, Faculdade de Farmácia, Universidade Federal de Minas Gerais, Av. Olegário Maciel, 2360, 30180 -112 Belo Horizonte - MG

Délio S. Raslan

Departamento de Química, Instituto de Ciências Exatas, Universidade Federal de Minas Gerais, Av. Antônio Carlos, 6627, 31270-010 Belo Horizonte - MG

\section{Hildebert Wagner}

Institut für Pharmazeutische Biologie der Universtität München, D-81377 München - Germany

Recebido em 23/9/03; aceito em 18/6/04; publicado na web em 8/10/04

CHEMICAL CONSTITUENTS AND CHROMATOGRAPHIC PROFILE OF THE STEM BARK OF Aspidosperma parvifolium A. DC. ("PAU-PEREIRA"). Four known alkaloids, uleine, epiuleine, apparicine and desmethyluleine, besides the isoprenoids lupeol and stigmasterol were isolated from the stem bark of Aspidosperma parvifolium. Their identification was based on spectroscopic analysis (UV, IR, ${ }^{1} \mathrm{H}-\mathrm{NMR},{ }^{13} \mathrm{C}-\mathrm{NMR}, \mathrm{MS}$ ). The chromatographic profile of the ethanolic extract was obtained by HPLC and uleine, epiuleine and apparicine were identified in the extract.

Keywords: Aspidosperma parvifolium; uleine; HPLC.

\section{INTRODUÇÃO}

A família Apocynaceae possui cerca de 200 gêneros e 2000 espécies, de distribuição tropical e subtropical, podendo chegar até regiões temperadas. O gênero Aspidosperma Mart. ocorre desde o México até a Argentina ${ }^{1}$ e distingue-se pela ocorrência freqüente de alcalóides indólicos ${ }^{2}$.

Algumas espécies de Aspidosperma são usadas popularmente como remédios para febre, mas $A$. parvifolium, apesar de ser citada como planta medicinal ${ }^{3}$, pouco se conhece sobre seu uso popular. É uma árvore com altura em torno de 10-15 m, com o tronco de 40-60 $\mathrm{cm}$ de diâmetro, que ocorre desde o sul da Bahia até o Rio Grande do Sul, em Minas Gerais, Goiás e no Mato Grosso. É conhecida popularmente como "guatambu", "peroba" ou "pau-pereira"4.

Desta espécie foi isolado, anteriormente, o alcalóide $\mathrm{N}$ metiltetraidroelipiticina ${ }^{2}$.

Este artigo descreve o estudo fitoquímico desta espécie, o isolamento e a caracterização do triterpeno lupeol, do esteróide estigmasterol e dos alcalóides uleína (1a), epiuleína (2), aparicina (3) e desmetiluleína (1b). Todos os constituintes químicos estão sendo descritos pela primeira vez em A. parvifolium.

Foram obtidos os perfis cromatográficos do extrato etanólico das cascas, através de CLAE, assim como dos alcalóides isolados e estes, identificados no cromatograma pelo tempo de retenção e espectros UV obtidos "on line".

\section{RESULTADOS E DISCUSSÃO}

O fracionamento cromatográfico do extrato etanólico das cascas de A. parvifolium revelou a presença de dois isoprenóides, lupeol e estigmasterol, e quatro alcalóides (Figura 1), uleína (1a), epiuleína (2), aparicina (3) e desmetiluleína (1b) que foram identificados atra-

*e-mail: lisieux@dedalus.lcc.ufmg.br

vés da análise dos dados fornecidos pelos espectros UV, IV, RMN ${ }^{1} \mathrm{H}$ $\mathrm{e}^{13} \mathrm{C}$ e de massas, comparados com valores registrados na literatura ${ }^{5-15}$.

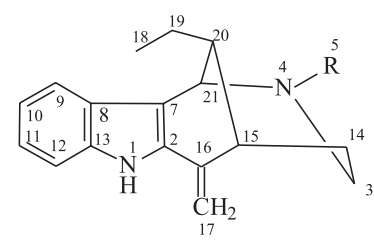

1a $\mathrm{R}=\mathrm{CH}_{3}$ uleína 1b $\mathrm{R}=\mathrm{H}$ desmetiluleína

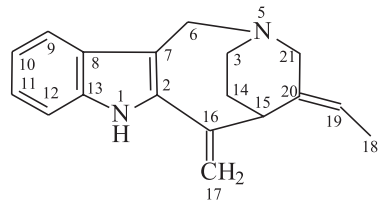

3

Figura 1. Alcalóides isolados (1-3) do extrato etanólico das cascas de A. parvifolium

Os espectros de ultravioleta (UV), obtidos "on line", em CLAE (acetronitrila:água), para uleína, epiuleína e aparicina foram muito parecidos, com máximos de absorção em 209, 240 e 309 nm (Figura 2) ${ }^{7}$.

Os espectros de massas, para uleína e epiuleína, exibem íons moleculares de $266 \mathrm{u}$. e fragmentos em $\mathrm{m} / \mathrm{z}, 237,223,209,194$ e 180 u., conforme proposta de Manske ${ }^{7}$. A aparicina exibe o íon molecular de 264 u., duas unidades de massa a menos que a uleína, com fragmentação muito semelhante. Quanto à desmetiluleína, o espectro de massas exibe o íon molecular de 252 u., ou seja, 14 unidades a menos que a uleína e fragmentação de acordo com aquela esperada para uleína ${ }^{8,9}$. 
Os espectros de $\mathrm{RMN}{ }^{1} \mathrm{H}$ para uleína e epiuleína são semelhantes, mas diferem em relação aos tripletos correspondentes aos grupos $\mathrm{CH}_{3}(\mathrm{C}-18)$ do grupo etila, centrados em $\delta 0,85$ para uleína e em $\delta 1,08$ para epiuleína. De acordo com a literatura, o grupo etila na uleína está sobre o sistema $\pi$-aromático e, portanto, espera-se para $3 \mathrm{H}-18$ um sinal mais próximo do TMS $(\delta 0,82)$, devido à proteção do sistema $\pi$, enquanto que na epiuleína o grupo etila está à direita e o mesmo sinal aparece em $\delta 1,08^{11}$.

$\mathrm{O}$ espectro de $\mathrm{RMN}{ }^{1} \mathrm{H}$ da aparicina difere daquele da uleína em dois aspectos principais: ausência do tripleto a $\delta 0,82$ atribuído aos três hidrogênios $3 \mathrm{H}-18$ da uleína e ausência de um simpleto a $\delta 2,30$ atribuídos aos 3 hidrogênios do grupo N-metila (3H-5) da uleína. Já o espectro de $\mathrm{RMN}{ }^{13} \mathrm{C}$ da aparicina difere do espectro da uleína, pela ausência dos sinais a $\delta 46,27$ e 24,35 , atribuídos respectivamente aos C-20 e C-19 da uleína. Outra diferença significativa foi o aparecimento de sinais de carbonos olefínicos a $\delta 131,39$ e 120,48.

Os espectros de $\mathrm{RMN}{ }^{13} \mathrm{C}$ da uleína, epiuleína e aparicina estão representados na Tabela 1 , com os valores dos deslocamentos químicos de ${ }^{13} \mathrm{C}$ para os três alcalóides e mostram completo acordo com as estruturas propostas ${ }^{12,13}$.

O espectro de massas do lupeol mostrou o pico do íon molecular em $\mathrm{m} / z, 426$ u. e do estigmasterol em $\mathrm{m} / z 412$ u. Os espectros de RMN ${ }^{1} \mathrm{H}$ dos dois sólidos mostraram acúmulos de sinais na faixa de $\delta$ 0,7 - 2,0 que, associados com o desenvolvimento de cor rosa com o teste de Lieberman-Buchard e ponto de fusão de 165 e $136{ }^{\circ} \mathrm{C}$, respectivamente, foram suficientes para identificação do lupeol e estigmasterol. A identificação foi confirmada pela fragmentação no espectrômetro de massas e pela análise comparativa de dados de RMN ${ }^{13} \mathrm{C}^{14,15}$.

O perfil cromatográfico do extrato etanólico das cascas de $A$. parvifolium mostrou um pico intenso com o tempo de retenção em 32 min que, comparado com os picos obtidos para a uleína (31,9 min) e epiuleína (31,4 min) e os espectros de UV “on line”, sugere tratar-se destes alcalóides (Figuras 2 e 3). Anterior a 32 min há um outro pico cujo tempo de retenção e o espectro UV "on line" que, comparado com aquele da aparicina (29,5 min), sugere tratar-se deste outro alcalóide (Figuras 2 e 3 ).

Não foi possível separar, nas condições cromatográficas utilizadas, a uleína da epiuleína, uma vez que cada um destes alcalóides
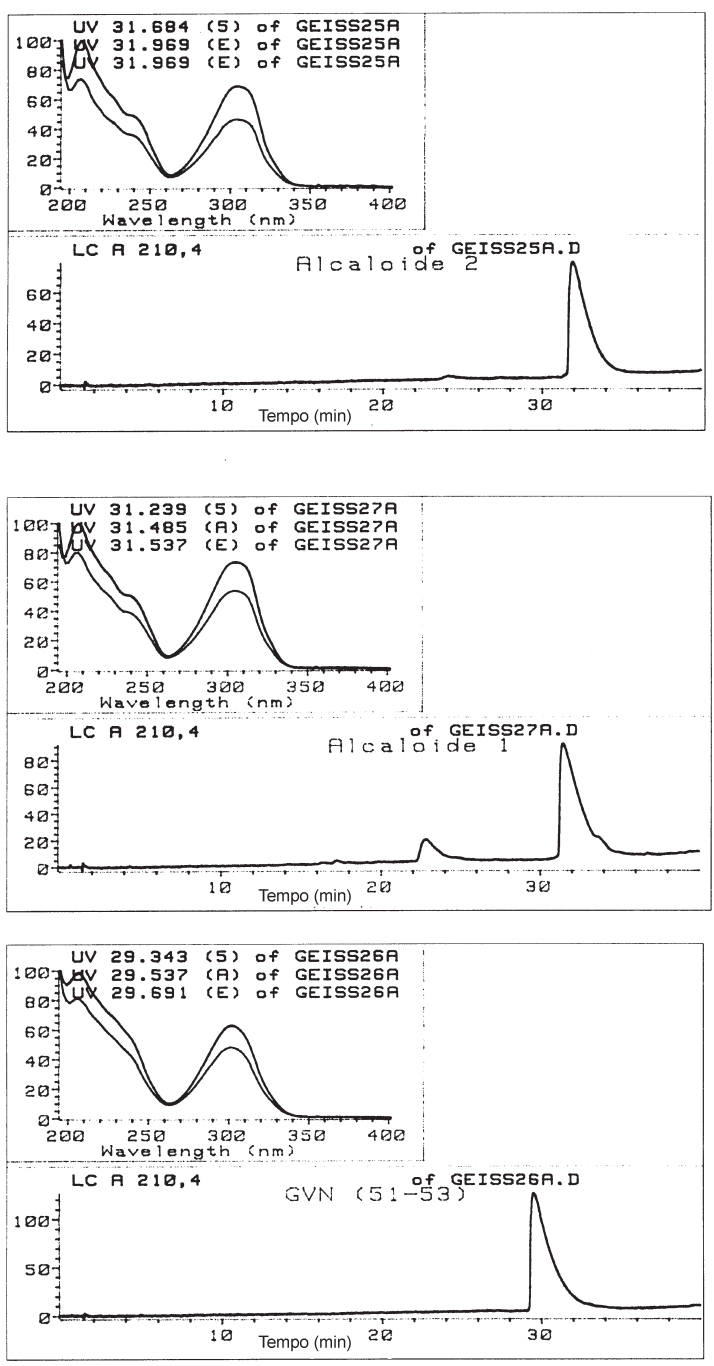

Figura 2. Cromatogramas a $210 \mathrm{~nm}$ e espectro UV "on line" da uleína, epiuleína e aparicina. Fase móvel: $\mathrm{ACN}-\mathrm{H}_{2} \mathrm{O}$ com 0,05\% de ac. hexanosulfônico, 10:90 - 90:10 em 50 min. Fluxo: $1 \mathrm{~mL} / \mathrm{min}$

Tabela 1. Deslocamentos químicos de ${ }^{13} \mathrm{C}(90 \mathrm{MHz})$ para uleína (1a), epiuleína (2) e aparicina (3) e comparação com a literatura (solvente $\mathrm{CDCl}_{3}$ )

\begin{tabular}{|c|c|c|c|c|c|c|}
\hline Carbonos & Uleína & Uleína ${ }^{12}$ & Epiuleína & Epiuleína $^{12}$ & Aparicina & Aparicina $^{13}$ \\
\hline 2 & 135,1 & 135,5 & 136,5 & 136,4 & 144,9 & 142,6 \\
\hline 3 & 46,3 & 46,2 & 46,4 & 46,2 & 45,2 & 45,3 \\
\hline 5 & 44,4 & 44,1 & 44,8 & 44,4 & & \\
\hline 6 & & & & & 54,1 & 54,3 \\
\hline 7 & 107,8 & 110,8 & 110,8 & 110,0 & 110,6 & 111,1 \\
\hline 8 & 129,4 & 129,3 & 128,5 & 127,9 & 128,9 & 129,0 \\
\hline 9 & 119,5 & 119,3 & 119,6 & 119,0 & 118,5 & 118,6 \\
\hline 10 & 119,8 & 119,6 & 119,9 & 119,8 & 122,9 & 123,0 \\
\hline 11 & 122,7 & 122,4 & 122,8 & 122,6 & 119,3 & 119,3 \\
\hline 12 & 110,7 & 110,8 & 111,9 & 111,0 & 110,3 & 110,2 \\
\hline 13 & 136,6 & 136,8 & 135,9 & 136,6 & 137,2 & 137,8 \\
\hline 14 & 34,7 & 34,5 & 28,5 & 27,8 & 29,4 & 29,6 \\
\hline 15 & 39,5 & 39,4 & 38,5 & 37,7 & 41,1 & 41,3 \\
\hline 16 & 138,7 & 138,7 & 142,1 & 141,1 & 135,6 & 135,7 \\
\hline 17 & 106,7 & 106,9 & 104,7 & 106,1 & 112,4 & 112,2 \\
\hline 18 & 11,8 & 11,6 & 12,2 & 12,0 & 12,6 & 12,5 \\
\hline 19 & 24,3 & 24,2 & 23,5 & 23,3 & 120,5 & 120,3 \\
\hline 20 & 46,1 & 45,8 & 44,7 & 44,2 & 131,4 & 131,3 \\
\hline 21 & 56,5 & 56,6 & 54,9 & 55,3 & 54,2 & 54,3 \\
\hline
\end{tabular}




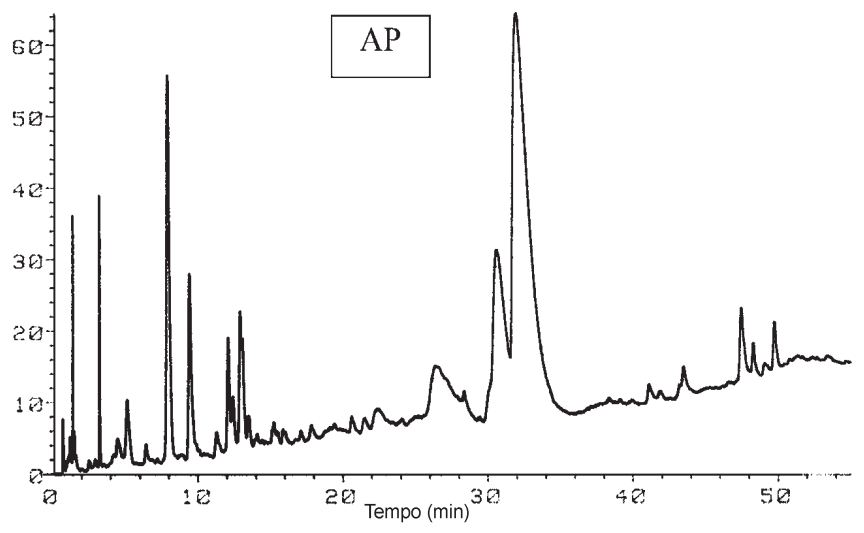

Figura 3. Cromatograma, obtido por CLAE, do extrato etanólico de A. parvifolium a $210 \mathrm{~nm}$. Fase móvel: $\mathrm{ACN}-\mathrm{H}_{2} \mathrm{O}$ com 0,05 de ác. hexanosulfônico, 10:90 - 90:10 em $50 \mathrm{~min}$. Fluxo: $1 \mathrm{~mL} / \mathrm{min}$

injetados separadamente, apresentou tempos de retenção muito próximos de 31,9 e 31,4 min, respectivamente (Figuras 2 e 3).

\section{PARTE EXPERIMENTAL}

\section{Material botânico}

As cascas de A. parvifolium foram coletadas em Paraobeba, Minas Gerais, e a espécie foi identificada pelo Dr. J. A. Lombardi, do Departamento de Botânica, do Instituto de Ciências Biológicas, UFMG, Belo Horizonte, MG.

\section{Extração e separação}

O extrato etanólico de A. parvifolium foi obtido por percolação a partir de $2,5 \mathrm{~kg}$ das cascas da árvore. Em torno de $60 \mathrm{~g}$ do extrato foram dissolvidos em etanol e $\mathrm{HCl} 1 \mathrm{~N}$ e fracionados com diclorometano. Obteve-se assim uma camada orgânica neutra (APN), uma interface (API) e uma camada aquosa ácida (APA). A camada aquosa ácida foi alcalinizada com $\mathrm{NH}_{4} \mathrm{OH}$ até $\mathrm{pH} 9$ e foi extraída com diclorometano (APT). Formou-se um precipitado que foi separado (APP). Este procedimento está sumariado na Figura 4.

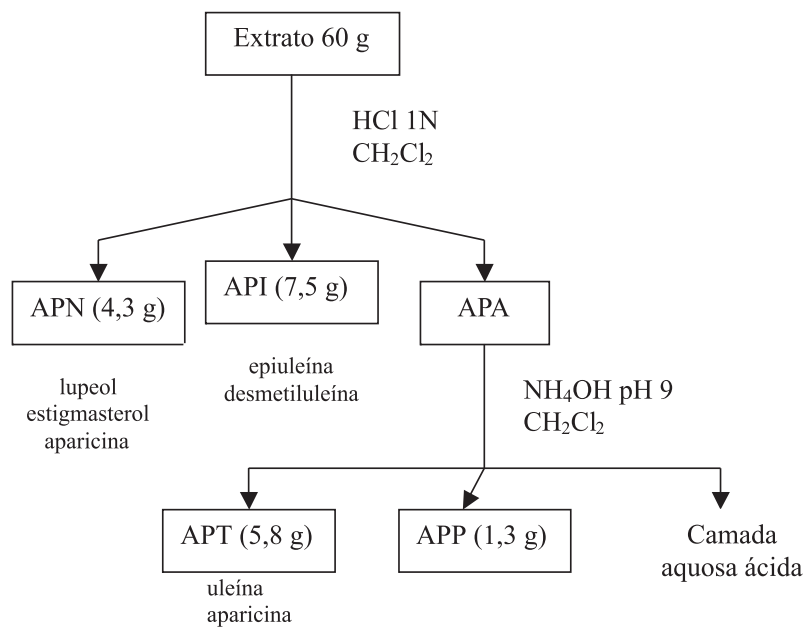

Figura 4. Fracionamento do extrato etanólico das cascas de A. parvifolium

\section{Cromatografia da fração neutra (APN 4,3 $\mathrm{g})$}

Esta fração foi eluída, em uma coluna de sílica-gel, com os eluentes em ordem crescente de polaridade. Das frações 6-10
$(0,124 \mathrm{~g})$ e 11-20 (0,119 g), através de CCD preparativa, usando-se como eluente de desenvolvimento tolueno-acetato de etila (93:7), foram isolados dois sólidos identificados como lupeol e estigmasterol, respectivamente.

Das frações 50-56 (0,178 g) e 61-63 (0,178 g), através de CCD preparativa, usando-se como eluente de desenvolvimento toluenoacetato de etila-dietilamina (7:2:1), foi isolado o sólido cristalino branco identificado como aparicina $(8,6 \mathrm{mg})$.

\section{Cromatografia da Interface (API 7,5 $\mathrm{g}$ )}

Este material foi fracionado em uma coluna de sílica-gel com os eluentes em ordem crescente de polaridade. As frações 59-60 (0,100 g) foram cromatografadas em CCD preparativa e foram isolados dois alcalóides: a desmetiluleína $(3,8 \mathrm{mg})$ e o alcalóide 6 (1 mg) que não teve sua estrutura elucidada.

\section{Cromatografia da camada orgânica (APT 5,8 g)}

A camada orgânica foi submetida a fracionamento em coluna de sílica-gel. As frações 5-20 (0,243 g) foram submetidas à CCD preparativa, usando-se como eluente clorofórmio:dietilamina (9:1). Obtiveram-se 9,2 mg do alcalóide identificado como epiuleína.

As frações 30-36 (0,542 g) foram recromatografadas em coluna de sílica-gel da qual foi isolado um sólido branco identificado como uleína (12 mg).

\section{Perfil cromatográfico e identificação por CLAE dos alcalóides} isolados nas cascas de $A$. parvifolium

Instrumentação e parâmetros de separação em CLAE

As análises foram feitas em um cromatógrafo líquido HP 1090 com detector de diodo-array HP 1040 em coluna de fase reversa de sílica RP-18 $(12,5 \mathrm{~cm}$ x $1 \mathrm{~cm})$ usando como fase móvel um gradiente linear de acetonitrila (ACN) com ac. hexanosulfônico e água (5$90 \%$ de acetonitrila) em $50 \mathrm{~min}$; fluxo $1 \mathrm{~mL} / \mathrm{min}$ e detecção no UV a $210 \mathrm{~nm}$.

Preparação das substâncias de referência

As substâncias de referência foram obtidas do extrato etanólico, conforme o esquema da Figura 4. Para injeção no cromatógrafo foram preparadas soluções de $1,0 \mathrm{mg} / \mathrm{mL}$ de cada um dos alcalóides isolados uleína, epiuleína e aparicina. Foram injetados no cromatógrafo $5 \mu \mathrm{l}$ de cada uma das soluções. Na Figura 2 estão representados os cromatogramas obtidos por CLAE e os espectros no UV obtidos "on line" para estes alcalóides.

\section{Preparação dos extratos para injeção em CLAE}

Três g da casca de $A$. parvifolium pulverizada foram extraídas em Soxhlet, por $12 \mathrm{~h}$, com etanol. O extrato etanólico foi concentrado a vácuo, fornecendo um resíduo de $0,649 \mathrm{~g}$. O resíduo foi dissolvido em acetonitrila e filtrado, completando o volume para um balão volumétrico de $5 \mathrm{~mL}$. Uma parte deste extrato foi novamente filtrado em Sep Pak RP-18 e $5 \mu$ foram injetados em CLAE. O cromatograma do extrato etanólico está representado na Figura 3.

Dados físico-químicos das substâncias isoladas das cascas de $A$. parvifolium

Uleína (1): Sólido cristalino branco: faixa de pf $78-85{ }^{\circ} \mathrm{C}$ em $\mathrm{CH}_{2} \mathrm{Cl}_{2}\left(75-100{ }^{\circ} \mathrm{C}\right)^{5}$. UV ( $\lambda$ max., nm): 209,240 e 309 em CLAE em acetonitrila/água (Figura 2). IV (vmax., $\left.\mathrm{KBr}, \mathrm{cm}^{-1}\right)$ : 3040, 1620, 870, 730. EM m/z: 266 u (M+100\%), 237 (20), 223(12), 209 (42), 194 (14), 180 (12). $\mathrm{RMN}^{1} \mathrm{H}\left(\mathrm{CDCl}_{3}, 360 \mathrm{MHz}\right) \delta: 0,85(3 \mathrm{H}, \mathrm{t}$, $\mathrm{J}=7,4 \mathrm{~Hz}, 3 \mathrm{H}-18) ; 1,15$ (2H, q, J=7,4Hz, 2H-19); 1,7 (H, m, H-20); 
2,05 (2H, m, 2H-14); 2,30 (3H, s, 3H-5); 2,7 (H, d, J=3,0 Hz, H-3); 2,45 (H, m, H-3); 3,5 (H, s, H-15); 4,10 (H, d, J=3Hz, H-21); 5,0 (H, s, H-17); 5,27 (H, s, H-17); 7,55 (H, d, J=8Hz, H-12); 7,35 (H, d, $\mathrm{J}=8 \mathrm{~Hz}, \mathrm{H}-9) ; 7,2$ (H, t, J=7,8Hz, H-11); 7,1 (H, t, J=7,8Hz, H-10); 8,25 (H, s, H-1).

Epiuleína (2): Sólido amorfo amarelo: pf $122-124^{\circ} \mathrm{C} \mathrm{em} \mathrm{CH}_{2} \mathrm{Cl}_{2}$ $\left(122-129^{\circ} \mathrm{C}\right)^{10}$. UV ( $\lambda$ max., nm): 209, 240 e 309 em CLAE em acetonitrila/água (Figura 2). EM m/z: 266 u (M+ 100\%), 237 (58); 223 (30); 209 (72); 194 (60); 180 (60); 167 (25). RMN'1 $\mathrm{H}\left(\mathrm{CDCl}_{3}\right.$, $360 \mathrm{MHz}) \delta: 1,08$ (3H, t, J=7,4 Hz, 3H-18); 1,25 (2H, t, 2H-19); 2,0 (2H, m, 2H-14); 2,25 (3H, s, 3H-5); 2,4 (H, m, H-3); 2,65 (H, m, H3); 4,0 (H, s, H-21); 4,95 (H, s, H-17); 5,2 (H, s, H-17); 7,3 (H, d, $\mathrm{J}=7,8 \mathrm{~Hz}, \mathrm{H}-9)$; 7,55 (H, d, J=8,0 Hz, H-12); 7, 15 (H, t, J=8,0 Hz, H11); 7,1 (H, t, H-10); 8,25 (H, s, H-1).

Aparicina (3): Sólido branco cristalino: pf $188-192^{\circ} \mathrm{Cem} \mathrm{CH}_{2} \mathrm{Cl}_{2}$ $\left(192-194{ }^{\circ} \mathrm{C}\right)^{8}$. UV ( $\lambda$ max., nm): 209, 240 e 309 nm em CLAE em acetonitrila/água (Figura 2). EM m/z: 264 u (M+100\%), 249 (20); 235 (28); 222 (28); 208 (37); 207 (20); 194 (17). RMN' ${ }^{1} \mathrm{H}\left(\mathrm{CDCl}_{3}\right.$, $360 \mathrm{MHz}) \delta: 1,47$ (3H, d, J=1Hz, 3H-18); 1,9 (2H, m, 2H-14); 3,98 (H, s, H-15); 4,27 (H, d J=17,7Hz, H-6); 4,47 (H, d, J=17,7Hz, H6); 5,3 (H, s, H-17); 5,4 (H, s, H-17); 5,3 (H, s, H-19); 3,8 (H, d, $\mathrm{J}=16 \mathrm{~Hz}, \mathrm{H}-21) ; 3,2$ (H, d, J=16Hz, H-21), 3,1(H, m, H-3); 3,4 (H, m, H-3); 7,3 (H, d, J=8Hz, H-9); 7,4 (H, m, J=8Hz, H-12); 7,2 (H, t, $\mathrm{J}=7 \mathrm{~Hz}, \mathrm{H}-10) ; 7,1(\mathrm{H}, \mathrm{t}, \mathrm{J}=7 \mathrm{~Hz}, \mathrm{H}-11) ; 7,95$ (H, s, H-1).

Desmetiluleína (1b): Sólido branco: pf $143{ }^{\circ} \mathrm{C} \mathrm{em} \mathrm{CH}_{2} \mathrm{Cl}_{2}{ }^{8}$. EM m/z: 252 u. (M+100\%), 223 (34); 209 (20); 194 (23); 180 (20). $\mathrm{RMN}^{1} \mathrm{H}\left(\mathrm{CDCl}_{3}, 360 \mathrm{MHz}\right) \delta: 0,85(3 \mathrm{H}, \mathrm{t}, \mathrm{J}=7,4 \mathrm{~Hz}, 3 \mathrm{H}-18) ; 1,15$ (2H, m, 2H-19); 1,65 (H, m, H-20); 1,85 (H, s, H-5); 2,05 (2H, m, 2H-14); 2,6 (H, d, H-15); 2,75 (H, d, J=2,8 Hz, H-3); 2,65 (H, m, H3); 5,3 (H, s, H-17); 5,0 (H, s, H-17); 4,4 (H, d, J=2,2 Hz, H-21); 8,2 (H, s, H-1); 7,55 (H, d, J=7,8 Hz, H-12); 7,45 (H, d, J=7,8 Hz, H-9); 7,1 (H, t, J=8 Hz, H-10); 7,2 (H, t, J=8 Hz, H-11).

Lupeol (APN - F 6-10): Sólido branco: pf 165-170 ${ }^{\circ} \mathrm{C}(160-$ $162{ }^{\circ} \mathrm{C}$ ). IV (vmax., $\mathrm{KBr}, \mathrm{cm}^{-1}$ ): 3340, 2940, 2860, 1640. EM m/z: 426 u. $\mathrm{RMN}^{1} \mathrm{H}\left(\mathrm{CDCl}_{3}, 360 \mathrm{MHz}\right) \delta: 0,8-1,8\left(\mathrm{CH}_{2}, \mathrm{CH}_{3}\right) ; 4,55(\mathrm{H}$, s, H-29); 4,65 (H, s, H-29); 3,6 (H, m, H-3). RMN ${ }^{13} \mathrm{C}\left(\mathrm{CDCl}_{3}, 360\right.$

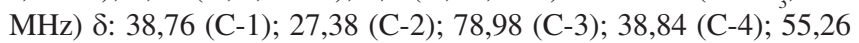
(C-5); 18,30 (C-6); 34,24 (C-7); 40,80 (C-8); 50,40 (C-9); 37, 14 (C10); 20,90 (C-11); 25,10 (C-12); 38,01 (C-13); 42,80 (C-14); 27,42 (C-15); 35,56 (C-16); 43,00 (C-17); 48,26 (C-18); 47,97 (C-19); 150,98 (C-20); 29,82 (C-21); 40,00 (C-22); 28,07 (C-23); 15,36 (C-
24); 16,11 (C-25); 15,95 (C-26); 14,53 (C-27); 17,99 (C-28); 109,31 (C-29); 19,29 (C-30) ${ }^{15}$.

Estigmasterol (APN - F 11-20): Sólido branco: pf $136{ }^{\circ} \mathrm{C} . \mathrm{EM}$ $m / z: 412$ u, 397 (70\%), 383 (100\%), 351 (9\%), 300 (14\%), 273 (19\%), 255 (40\%), $213(7 \%) . \mathrm{RMN}^{1} \mathrm{H}\left(\mathrm{CDCl}_{3}, 360 \mathrm{MHz}\right) \delta: 0,7-2,0\left(\mathrm{CH}_{2}\right.$, $\left.\mathrm{CH}_{3}\right) ; 3,5(\mathrm{H}, \mathrm{m}, \mathrm{H}-3) ; 5,0(\mathrm{H}, \mathrm{m}) ; 5,3(\mathrm{H}, \mathrm{m}) ; 5,4(\mathrm{H}, \mathrm{s}) . \mathrm{RMN}^{13} \mathrm{C}$ $\left(\mathrm{CDCl}_{3}, 360 \mathrm{MHz}\right) \delta:$ 37,22 (C-1); 31,62 (C-2); 71,79 (C-3); 42,25 (C-4); 140,72 (C-5); 121,7 (C-6); 31,87 (C-7); 31,87 (C-8); 50,10 (C-9); 36,48 (C-10); 21,06 (C-11); 39,74 (C-12); 42,19 (C-13); 56, 84 (C-14); 24,34 (C-15); 28,91 (C-16); 56,02 (C-17); 12,03 (C-18); 19,38 (C-19); 40,49 (C-20); 21,06 (C-21); 138,31 (C-22); 129,24 (C-23); 51,21 (C-24); 31,87 (C-25); 21,20 (C-26); 18,96 (C-27); 25,39 (C-28); 12,24 (C-29) $)^{14}$.

\section{AGRADECIMENTOS}

Ao KFA Deutsches Forschungszentrum Juelic GmbH pela bolsa de estudos e ao CNPq pela passagem concendida.

\section{REFERÊNCIAS}

1. Ferreira Neto, W; M.; Tese de Doutorado, Universidade Estadual de Campinas, Brasil, 1988.

2. Bolzani, L.; Serur, L. M.; Matos, F. J. A.; Gottlieb, O. R.; Biochem. Syst. Ecol. 1987, 15, 187.

3. Correa, M. P.; Dicionário das plantas úteis do Brasil, Ministério da Agricultura: Rio de Janeiro, 1984, vol. 5, p.451.

4. Lorenzi, H.; Árvores brasileiras. Manual de identificação e cultivo de plantas arbóreas nativas no Brasil, Ed. Plantarum: São Paulo, 1992, p. 24.

5. Büchi, G.; Warnhoff, E. W.; J. Am. Chem. Soc. 1959, 81, 4433.

6. Ohashi, M.; Joule, J. A.; Djerassi, C.; Tetrahedron Lett. 1964, 51, 3899.

7. Manske, R. H. F.; Rodrigo, R.; The alkaloids, Academic Press: New York. 1965, vol.8.

8. Joule, J. A; Monteiro, H.; Durham, L. J.; Gillbert, B; Djerassi, C.; J. Chem. Soc. 1965, 4773.

9. Joule, J. A.; Ohashi, M.; Gilbert, B.; Djerassi C.; Tetrahedron 1965, 21, 1717.

10. Gaskell, A. J.; Joule, J.; Chem. Ind. 1967, 24, 1089.

11. Büchi, G.; Gould, S. J.; Näf, F.; J. Am. Chem. Soc. 1971, 93, 2492.

12. Borris, R. P.; Lankin, D. C.; Cordell, G.; J. Nat. Prod. 1983, 46, 200.

13. Massiot, G.; Zeches, M.; Thépenier, P.; Jacquier, M- J.; Le Men-Olivier, L.; Delaude, C.; J. Chem. Soc. Chem. Commun. 1982, 768.

14. Dey, P. M.; Harbone, J. B.; Methods in plant biochemistry terpenoids, Academic Press: London, 1991, vol. 7.

15. Mahato, S. B.; Kundu, A. P.; Phytochemistry 1994, 37, 1517. 